\title{
Vehicle Scheduling of the Emergency Medicines in the Early Period After Earthquake Disaster
}

\author{
Li Dawei ${ }^{1, *}$, Xing Yingying ${ }^{1}$ and Wang $\mathrm{Li}^{2}$ \\ ${ }^{1}$ School of Science, University of Science and Technology Liaoning, Anshan, Liaoning, 114051, P.R. China; ${ }^{2}$ School of \\ Electronic and Information Engineering, University of Science and Technology Liaoning, Anshan, Liaoning, 114051, \\ P.R. China
}

\begin{abstract}
In this paper, based on the analysis of the time urgency in medicine relief demand and kinds of complexity in the early period after earthquake disasters, the problem about vehicle scheduling in the medicine dispatching process is studied. A mathematical model of vehicle scheduling in the medicine dispatching process is built, which objective function is to minimize the total transportation time, under the condition of single depot, multi-type vehicles and multiconstraints. The solutions are obtained by using genetic algorithm based on natural number encoding. The impacts of different parameters on the results have been compared by software Matlab. The experiment results show that genetic algorithm is satisfactory to solve this problem.
\end{abstract}

Keywords: Early period after earthquake disaster, genetic algorithm, natural number encoding, vehicle scheduling.

\section{INTRODUCTION}

Like desertification, flood, tsunamis, typhoon, dust storm and other disasters, earthquake is one of the global common natural disasters. Its unexpected and highly destructive often cause significant casualties. Characteristics of earthquake disaster are:

\section{- High Frequency}

In Chinese continent, for example, more than 3000 times earthquake activities with Magnitude $M s \geq 5$ occurred from last century to now, among them there were more than 800 times with Magnitude $M s \geq 6$, and even more than 11 times with Magnitude $M s \geq 8$.

\section{- High Magnitude}

It is reported that 13 times earthquake activities with Magnitude $M s \geq 8$ occurred in global since this century, among them 2 times occurred in China. And

\section{- Wide Distribution}

According to statistics, earthquake activities with Magnitude 5 had occurred in all provinces, autonomous regions and municipalities in China.

Most countries in the world have established emergency response mechanism. For examples, The United States is a country with natural disasters high frequency. Federal Emergency Management Agency (FEMA) was set up in 1979, and Federal Emergency Response Plan officially launched in the nineties of the last century. Article 22 in China's The Act of Destructive Earthquake Emergency stipulates: After the destructive earthquake, the emergency period is generally 10 days; if necessary, it may be extended by 20 days.

After the earthquake disaster, the timely rescue of the wounded is very important. The timely supply and transporttation of emergency medicines after disaster are essential to save lives. Usually emergency medicines are dispersedly stored in the administrative departments or major medical departments. Once disaster occurs, emergency medicines are dispatched to the disaster areas ordered by the competent authorities, Centers for Disease Control (CDC), for example. Transportation of emergency medicines is vital in the lives of the people in the disaster areas.

Currently, with little research specifically on emergency medicines logistics in earthquake activities, this paper is mainly to study the vehicle scheduling problem of emergency medicines dispatching in the early period after earthquake disaster. Because of different earthquakes in the same area and different extents of the damage in different parts of the same earthquake, emergency period is deter-mined according to the actual progress of the rescue. Such as $\mathrm{Xu}$ Yong, who involved in Wenchuan earthquake activity with Magnitude $M s=8.0$ in May 12, 2008, summarized the kinds of medicines demand of each period after the earthquake [1]. Earthquake disaster emergency period may be divided into three periods: 1-2 weeks early; 2-3 weeks for the mid-term; and 3-4 weeks later [2]. The early is mainly in trauma patients accounted for about $72 \%$. Here, according to the different needs of the rescue mission, the devastating earthquake emergency period is divided into two periods: (1) 1-3 days after earthquake, the main task is to search stranded people and rescue the wounded; and (2) 4-10 days after earthquake, the main task is the treatment of the injured and 
disease prevention. In each period, with the differences of disease, the kinds of medicines, the demands for different medicines throughout the disaster, transportation requirements change over time. While the actual scheduling of medicines, often with medicines and other supplies together, ignores hierarchical demands and specific requirements on the transportation of specific medicines, which leads to some medicines failure and loss, delays treating the injured, and more generates unnecessary waste.

In order to solve these problems, such that disaster relief emergency medicines can play the biggest role, maximize the treatment of the wounded and reduce casualties, the paper devotes to the analysis of the specific needs of vehicle scheduling of emergency medicines in the early period after earthquake, and designs the vehicle scheduling model of closing to the actual situation of emergency medicines in the early period after earthquake, which is solved with genetic algorithms.

\section{DESCRIPTION AND MODELING}

\subsection{Problem Description}

After the earthquake disaster, government departments must immediately organize rescue work. A lot of relief supplies, including medicines, may be transferred to the disaster area from all directions. In the early period after earthquake disaster, because of more trauma patients, about 30 kinds of major medicines are needed, including analgesics, antiinfective, haemostatic, electrolytes, water and acid-base balance of the drugs, as well as some auxiliary drugs to promote wound healing, nutrition body and nerve cells, and some drugs to treat of skin allergies, digestive, fever, and other diseases. Among them, cold drugs, antibiotic drugs are easily added, while drugs of digestive system, ophthalmology, dermatology and trauma are relatively scarce. The extensive use of anesthesia, nerve and disinfection, anti-virus drugs in the late should also have reserves $[3,4]$.

Because of wide kinds of drugs, and different drugs have different requirements on the ambient temperature, relative humidity and freshness. Such as serum, insulin, and enzymes preparations need cryopreservation. Special injections require sterile storage. Narcotic drugs will turn bad if exposed to light, so must be stored in the dark. In addition, the disaster area is likely to more than one, and the affected locations scattered. With the demands, the kinds of drugs and the special requirements of the vehicle will be studied to solve this problem in this paper.

\subsection{Modeling Assumptions}

As described above, this multi-area, multi-vehicle scheduling problem is very difficulty to build model. In order to solve the problem in a more practical manner, some assumptions about the problem must be made [5]. Here assume that:

(1) The vehicles departure from the medicine reservation center, after a series of affected locations return to the reservation center.

(2) The distance between any two affected locations or the distance from the medicine reservation center to any one affected location is as a known.
(3) For the multi-type vehicles, the load capacity and speed of various types are known.

(4) Drug inventory of medicines reservation center will meet the needs of the affected locations, while the demand for single location will be less than the maximum load of the vehicle.

(5) All road conditions are ideal, without considering the impact on the speed of the vehicle and the unloading time.

\subsection{Symbol Description}

In order to convenience modeling, some mathematical symbols are used below.

Medicine reservation center is numbered by 0 ; the affected locations are numbered by $1,2, \cdots, n$, noted by $a_{i}$; The vehicle types are numbered by $1,2, \cdots, m$, noted by $k$.

$b_{k} \quad$ number of vehicle type $k$

$u_{k} \quad$ loading capacity of vehicle type $k$

$v_{k} \quad$ driving speed of type $k$

$r_{i} \quad$ demand of $a_{i}$

$\omega_{i} \quad$ vehicle arrives at $a_{i}$

$h_{i} \quad$ the latest time allowed the vehicle to reach $a_{i}$

$d_{i j} \quad$ distance of $a_{i}$ to $a_{j}$

Define the decision variables as below:

$x_{i j}^{k s}= \begin{cases}1, & \text { veihcle } s \text { of type } k \text { from } a_{i} \text { to } a_{j} ; \\ 0, & \text { else. }\end{cases}$

$y_{i}^{k s}= \begin{cases}1, & r_{i} \text { is completed by vehicle } s \text { of type } k \\ 0, & \text { else. }\end{cases}$

\subsection{Model Construction}

According to the above assumptions, problem mathematical model can be constructed as below:

$$
\begin{aligned}
& \min t=\sum_{k=1}^{m} \sum_{s=1}^{b_{k}} \sum_{i=0}^{n} \sum_{j=0}^{n} \frac{x_{i j}^{k s} d_{i j}}{v_{k}} \\
& \text { s.t. } \sum_{i=1}^{n} r_{i} y_{i}^{k s} \leq u_{k}, \forall k, s
\end{aligned}
$$

$\sum_{k=1}^{m} \sum_{s=1}^{b_{k}} y_{i}^{k s}=1, i=1,2, \cdots, n$

$\sum_{j=0}^{n} x_{i j}^{k s}=y_{i}^{k s}, i=1,2, \cdots, n ; \forall k, s$

$\sum_{i=0}^{n} x_{i j}^{k s}=y_{j}^{k s}, j=1,2, \cdots, n ; \forall k, s$ 


$$
\begin{aligned}
& \sum_{k=1}^{m} \sum_{s=1}^{b_{k}} \sum_{i=0}^{n} x_{i 0}^{k s}=\sum_{k=1}^{m} \sum_{s=1}^{b_{k}} \sum_{j=0}^{n} x_{0 j}^{k s} \\
& \omega_{j}=\sum_{i=0}^{n} \sum_{k=1}^{m} \sum_{s=1}^{b_{k}}\left(\omega_{i}+\frac{d_{i j}}{v_{k}}\right) x_{i j}^{k s}, j=1,2, \cdots, n \\
& \omega_{i} \leq h_{i}, i=1,2, \cdots, n
\end{aligned}
$$

\subsection{Model Explanation}

Objective function (1) represents the total shortest delivery time; constraint (2) represents each vehicle delivery task is no more than the vehicle's maximum loading capacity; constraint (3) represents each affected location has and only has a vehicle for the distribution; constraint (4) represents affected location for the distribution by the vehicle $s$ of type $k$, which must be sure to traffic into from other affected location, and exit from the affected location, then into the other affected locations; constraint (5) represents affected location for the distribution by the vehicle $s$ of type $k$ which must be sure to traffic into from other affected location, and exit from $a_{j}$, then into the other affected locations; constraint (6) represents the number sailed out of center equals to the number of returned vehicles; constraint (7) represents the moment of the vehicle $s$ of type $k$ arrives at $a_{j}$; constraint (8) represents the latest time of $a_{i}$ to access medicines.

\section{GENETIC ALGORITHM SOLUTION}

\subsection{Design Ideas}

The paper takes the traditional natural number encoding. In the chromosome, 0 represents medicine reservation center; $1,2, \cdots, n$ represent the demand locations; $m$ is the number of vehicle. Randomly arrange the $n+1$ demand locations, and inserted $m+1$ zeros into the number, such that the first and the end one are 0 , and the middle can not have two adjacent 0 . For example, chromosome:

$0,3,7,5,1,0,6,10,9,12,0,2,8,11,4,0$

expresses for 12 demand locations, 3 vehicles. The vehicles driving path are:

$$
\underbrace{0 \rightarrow 3 \rightarrow 7 \rightarrow 5 \rightarrow 1 \rightarrow 0}_{\text {path } 1}, \quad \underbrace{0 \rightarrow 6 \rightarrow 10 \rightarrow 9 \rightarrow 12 \rightarrow 0}_{\text {path } 2},
$$

and $\underbrace{0 \rightarrow 2 \rightarrow 8 \rightarrow 11 \rightarrow 4 \rightarrow 0}_{\text {path } 3}$

\subsection{Fitness Function}

It calculates the delivery time with the handling skills of multi-type vehicle and the formula (1). If the constraint condition is not satisfied, it is added a penalty term to the objective function.

\subsection{Genetic Operators}

\subsubsection{Selection Operator}

Roulette wheel selection strategy is taken as selection operator here. This selection strategy ensures that the better chromosome in the present generation survives in the next generation in a large probability [6].

\subsubsection{Crossover Operator}

In order to retain preferably adjacent relationship in cross operation, two parent chromosomes are selected to retain a path of the parent 1 and the number of affected locations in each path. The rest of affected locations are obtained from parent 2. Offspring 1 is obtained in accordance with the OX (Order Crossover) operation. Similarly, offspring 2 can be obtained. For example, parent $P_{1}$ and parent $P_{2}$ are:

$$
\begin{aligned}
& P_{1}=0,2,3,4,0,6,5,8,0,7,1,0 ; \\
& P_{2}=0,1,5,8,0,7,4,0,6,3,2,0 .
\end{aligned}
$$

If offspring reserves the path 1 of the parent $P_{1}$, then offspring is:

$$
O_{1}^{\prime}=0,2,3,4,0,1,5,8,0,7,6,0 \text {. }
$$

If offspring reserves the path 2 of the parent $P_{1}$, then offspring is:

$$
O_{1}^{\prime \prime}=0,1,7,4,0,6,5,8,0,3,2,0 \text {. }
$$

If offspring reserves the path 3 of the parent $P_{1}$, then offspring is:

$$
O_{1}^{\prime \prime \prime}=0,5,8,4,0,6,3,2,0,7,1,0 .
$$

The same method can be used to get offspring $\mathrm{O}_{2}$.

\subsubsection{Mutation Operator}

For a randomly choose path, reversion is adopted as mutation operation. Such as chromosome $P$ :

$P=0,2,5,3,0,8,1,4,0,7,6,0$.

Choose path 2 to mutate, then we have $P^{\prime}$ after mutation:

$P^{\prime}=0,2,5,3,0,4,1,8,0,7,6,0$.

\subsection{Termination Criterion}

Like most of the genetic algorithms, the number of itera-tion is taken as the termination criterion to stop searching.

\section{NUMERICAL EXAMPLE}

Assuming that there are one of type A, two of type B, noted A, B1 and B2 with load capacity 9, 7, 7, travel speed 50,60 , and $60 \mathrm{~km} / \mathrm{h}$, respectively. Eight affected locations must receive drugs from medicine reservation center. Termination condition is set the iteration number (generation number) $=100$.

Genetic algorithm parameter selection is very important for solving model [8]. The paper with the numerical example, fix other parameters unchanged and select it from the common range of the determine parameter by software Matlab programming.

The population size is determined in 20-200; the crossover probability is in $0.5-0.95$; and the mutation probability 


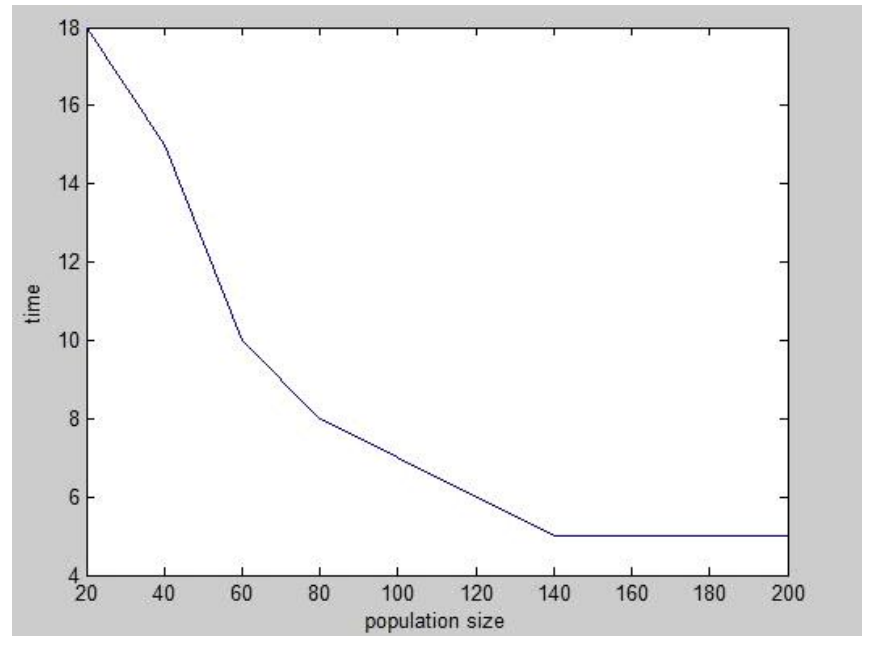

Fig. (1). Curve of time with population size.

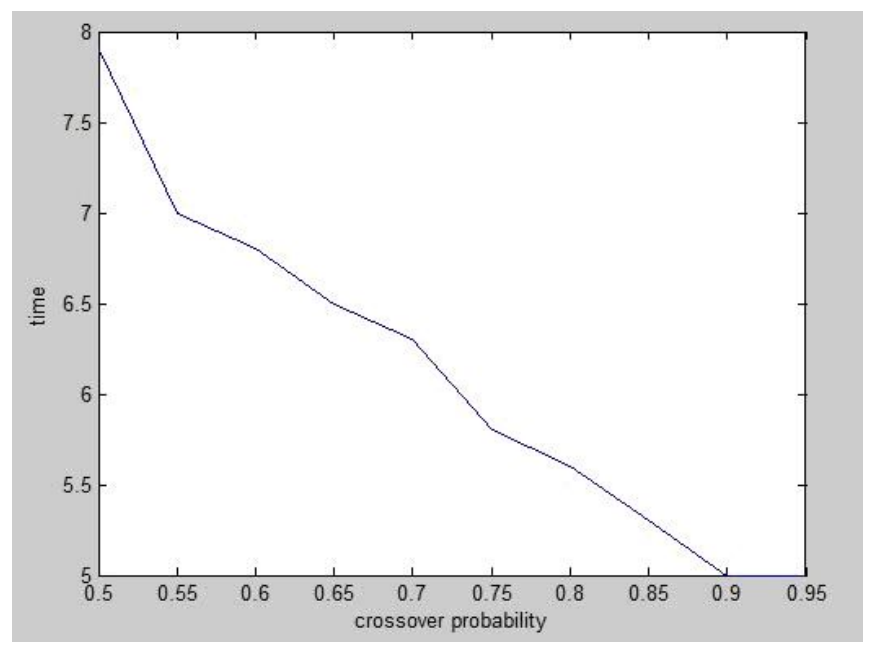

Fig. (2). Curve of time with crossover probability.

is in 0.005-0.05. It can be seen from Fig. (1), the optimal value is beginning to stabilize when population size is reached after 120 on the condition that other parameters are unchanged; the cross probability on the impact of the optimal value is very unstable.

However GA convergence reaches stabilize if crossover probability is 0.9 , see Fig. (2).

Mutation probability with minimal impact on the optimal value, the entire trend line has stabilized, this phenomenon can be seen from Fig. (3). So here take the population size of 120 , crossover probability of 0.95 , and mutation probability of 0.05 . Fig. (4) is a better result of the genetic algorithm convergence process graph with parameters selected above, which result is obtained after many operations using software Matlab in the example. Here, genetic algorithm converges to a better solution from a random initial solution. GA started from a random initial solution eventually converges to a better solution.

The final optimal solution: minitime $=5.0 \mathrm{~h}$. At this case, each vehicle without overloading arrives before the latest time allowed by the affected locations. The paths of vehicle $\mathrm{A}, \mathrm{B} 1$ and $\mathrm{B} 2$ are:

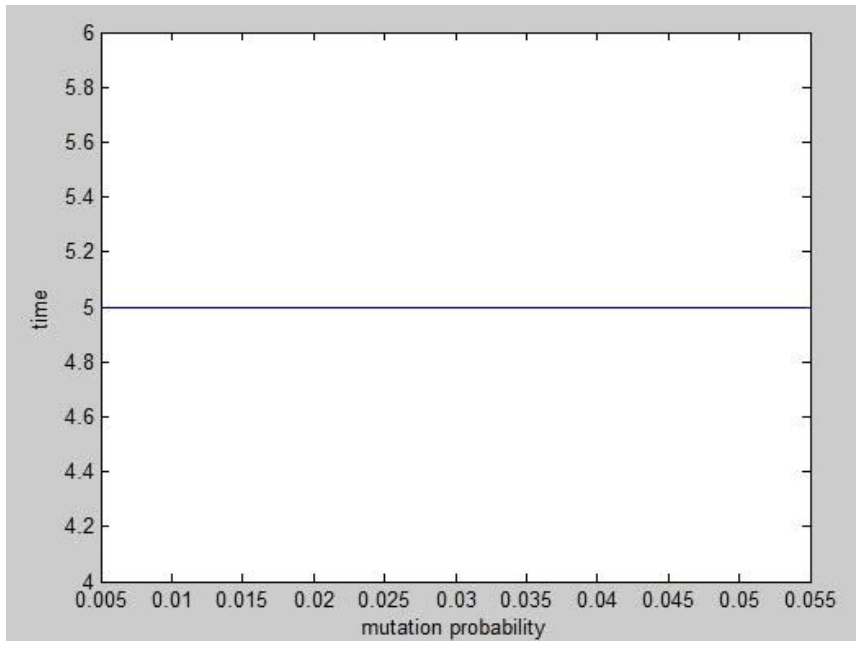

Fig. (3). Curve of time with mutation probability.

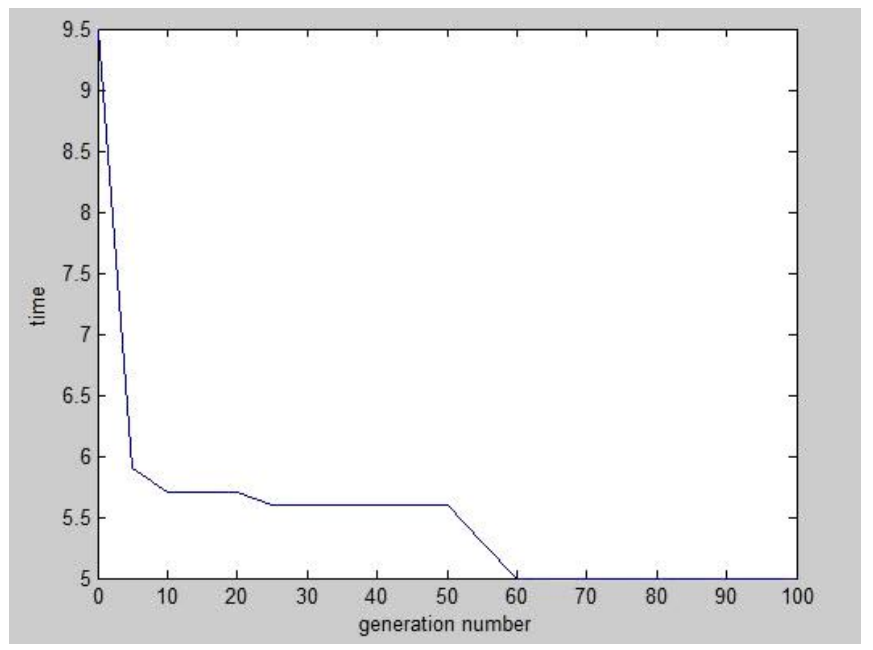

Fig. (4). Convergence process of genetic algorithm.

$$
\begin{aligned}
& \underbrace{0 \rightarrow 3 \rightarrow 5 \rightarrow 1 \rightarrow 0}_{\text {path } \mathrm{A}}, \quad \underbrace{0 \rightarrow 2 \rightarrow 7 \rightarrow 4 \rightarrow 0}_{\text {path } \mathrm{B} 1}, \quad \text { and } \\
& \underbrace{0 \rightarrow 6 \rightarrow 8 \rightarrow 0}_{\text {path B2 }} \\
& \text { respectively. }
\end{aligned}
$$

\section{CONCLUSION}

The initial earthquake relief is very important for the lives of the people in the disaster areas. According to the characteristics of commonly used drugs after earthquake early, the paper has established vehicle scheduling model for medicines in earthquake early. Genetic algorithm based on natural number "zero insertion" coding is used directly to prevent illegal chromosomes. In solving process, it combines with the data in the example, and use software Matlab to obtain a common range of different parameters. Eventually, the better parameters are selected to get the optimal solution after multiple calculations. This method has good idea and easy to operate. It contributes to the maximum extent possible to reduce the life-threatening brought by the devastating earthquake. 


\section{CONFLICT OF INTEREST}

The authors confirm that this article content has no conflict of interest.

\section{ACKNOWLEDGEMENTS}

This work was financially supported by the Natural Science Foundation of Liaoning Province (20092177 and 20102097).

\section{REFERENCES}

[1] Y. Xu, Q. Z. Wei, H. Meng, and S. Liu, "Thinking of field medical team medicine supply in earthquake relief", Pharmaceutical Care and Research, vol. 8, pp. 385-386, 2008.

[2] C. J. Yuan, J. P. Yu, J. Zhou, and W. Lin, "The application of emergency rescue drugs in the earthquake relief for public health emergencies", Chinese Journal of Modern Drug Applica-tion, vol. 2, pp. 117-119, 2008.
[3] H. J. Feng, J. W. Li, S. X. Chen, M. H. Liu, and X. H. Sun, "Demand analysis on drugs for earthquake casualty", The Journal of Pharmaceutical Practice, vol. 21, pp. 100-102, 2003.

[4] R. Tang, "Emergent medicine support in earthquake relief", Pharmaceutical Care and Research, vol. 8, pp. 401-403, 2008

[5] Z. J. Ye, H. Z. Ye, D. P. Zhou, and H. Y. Yi, "Heuristics for the fleet size and mix vehicle routing problem", Journal of Highway and Transportation Research and Development, vol. 22, pp. 147$151,2005$.

[6] B. L. Xie, J. Li, and Y. H. Guo, "Genetic algorithm for vehicle scheduling problem of non-full loads with time windows", Journal of System Engineering, vol. 15, pp. 290-294, 2000.

[7] Q. G. Lin, "Research of the Vehicle Routing Problem with Time Windows based on Hybrid Genetic Algorithm", M. S. Thesis, Shandong University, Ji'nan, Shandong, P. R. China, 2007.

[8] S. Lawrence, and A. Mohammad, "Parametric experimentation with a genetic algorithmic configuration for solving the vehicle routing problem", In: Proceedings of Annual Meeting of the Decision Sciences Institute, Las Vegas, 1996, pp. 488-490.

(C) Dawei et al.; Licensee Bentham Open.

This is an open access article licensed under the terms of the Creative Commons Attribution Non-Commercial License (http://creativecommons.org/licenses/by-nc/3.0/) which permits unrestricted, non-commercial use, distribution and reproduction in any medium, provided the work is properly cited. 